\title{
INTERACTING QUARTER-PLANE LATTICE WALK PROBLEMS: SOLUTIONS AND PROOFS
}

\author{
RUIJIE XUD \\ (Received 7 July 2021; first published online 5 November 2021)
}

2020 Mathematics subject classification: primary 05C81; secondary 05E99.

Keywords and phrases: lattice paths, random walk, quarter-plane, kernel method, algebraic generating functions, D-finite functions, homomorphisms on a Riemann surface.

Lattice walk problems in the quarter-plane have been widely studied in recent years. The main objective is to calculate the number of configurations, that is, the number of $n$-step walks ending at certain points or, alternatively, the generating function of the walks. In combinatorics, physics and probability theory, other properties such as asymptotic behaviour and the algebra of the generating functions are also of interest.

In this thesis we focus on solving quarter-plane lattice walks with interactions via the kernel method. We assign interaction $a$ to the $x$-axis, $b$ to the $y$-axis and $c$ to the origin. We denote $q_{n, k, l, h, u, v}$ as the number of $n$-step paths that start at point $(0,0)$ and end at point $(k, l)$ and which visit vertices on the horizontal boundary (except the origin) $h$ times, vertices on the vertical boundary (except the origin) $v$ times and the origin $u$ times. Then $Q(x, y, t)=\sum_{n, k, l, h, u, v} q_{n, k, l, h, u, v} x^{k} y^{l} t^{n} a^{h} b^{v} c^{u}$ is the generating function of the walk. Our aim is to find whether the interactions will affect the solubility of quarter-plane lattice walk models and the properties of the generating functions $Q(x, y, t)$. In particular, we give solutions to all 23 quarter-plane lattice walks associated with finite groups. We solve 21 of them explicitly, writing an expression for the generating functions $Q(x, y, t)$. The other two are only partly solved. We discuss when the generating function $Q(x, y, t)$ becomes D-algebraic, D-finite and algebraic by the variations of $a, b, c$.

We also compare the solutions obtained by the kernel method and the solutions obtained by an analytic method based on homomorphisms on a Riemann surface. We combine the solutions in another form with the solution of the kernel method and prove the properties of the generating function $Q(x, y, t)$. We observe that the solution obtained using the kernel method and other analytic methods are consistent with one another.

Some of this research has appeared in [1,2].

Thesis submitted to the University of Melbourne in March 2021; degree approved on 31 March 2021; supervisors Aleks Owczarek, Richard Brak and Nicholas Beaton.

(C) 2021 Australian Mathematical Publishing Association Inc. 


\section{References}

[1] N. R. Beaton, A. L. Owczarek and R. Xu, 'Quarter-plane lattice paths with interacting boundaries: Kreweras and friends', Sém. Lothar. Combin. 82B (2019), Article no. 26, 12 pages.

[2] N. R. Beaton, A. L. Owczarek and R. Xu, 'Quarter-plane lattice paths with interacting boundaries: the Kreweras and reverse Kreweras models', Transcendence in Algebra, Combinatorics Geometry and Number Theory, A. Bostan and K. Raschel (eds.), Springer Proceedings in Mathematics and Statistics, 373 (Springer, Cham, 2021), Chapter 7.

RUIJIE XU, School of Mathematics and Statistics, University of Melbourne,

Melbourne, Victoria 3010, Australia

e-mail: 710664016@qq.com 